\title{
Second Harmonic Scattering from Silver Nanocubes
}

Isabelle Russier-Antoine, ${ }^{1}$ Hye Jin Lee, ${ }^{2}$ Alastair W. Wark, ${ }^{3}$ Jérémy Butet, ${ }^{4}$ Emmanuel Benichou, ${ }^{1}$ Christian Jonin, ${ }^{1}$ Olivier J.F. Martin, ${ }^{4}$ Pierre-François Brevet ${ }^{1 *}$

${ }^{1}$ Institut Lumière Matière, UMR CNRS 5306, Université Claude Bernard Lyon 1, 10 Rue Ada Byron, F-69622 Villeurbanne cedex, France

${ }^{2}$ Department of Chemistry and Green-Nano Materials Research Center, Kyungpook National University, 1370 Sankyuk-dong, Buk-gu, Daegu-city, 702-701, Republic of Korea

${ }^{3}$ Centre for Molecular Nanometrology, Technology and Innovation Centre, Department of Pure and Applied Chemistry, 99 George Street, University of Strathclyde, Glasgow, G1 1RD, United Kingdom

${ }^{4}$ Nanophotonics and Metrology Laboratory (NAM), Swiss Federal Institute of Technology Lausanne (EPFL), CH-1015, Lausanne, Switzerland

\footnotetext{
* Corresponding Author : Prof. Pierre-François Brevet, pfbrevet@univ-lyon1.fr
} 


\begin{abstract}
:
The second harmonic light scattered from silver nanocubes dispersed in an aqueous suspension is investigated. The first hyperpolarizability is determined and corrected for resonance enhancement. It is shown to be similar to that of silver nanospheres with a comparable volume. The polarization-resolved analysis of the scattered harmonic intensity exhibits a surface response strongly modulated by the different multipolar field contributions. As a result, the shape does not play a leading role anymore for nanoparticles with a centrosymmetric shape when retardation must be considered. Comparing the right angle and forward-scattered polarized intensity responses, the unequal balance of the eight nanocube corners' contribution to the total response is revealed despite the high degree of centrosymmetry of the cubic shape. It is then demonstrated with a simple model that the nanocubes' first hyperpolarizability exhibits an octupolar tensorial symmetry. The surface integral equation method calculations are finally provided to investigate further the role of the corners' and edges' rounding.
\end{abstract}




\section{INTRODUCTION}

The investigation of the optical properties of noble metal nanoparticles has received considerable attention over the last decades owing to their potential application in a wide range of fields, from imaging and sensing to optoelectronics. ${ }^{1-7}$ This interest largely relies on the plasmonic properties of these metallic nanoparticles, especially gold and silver nanoparticles. Surface plasmon (SP) resonances associated with the collective oscillation of the conduction band electrons within the visible and the near infra-red parts of the optical spectrum provide an insightful tool to investigate these optical properties in particular. Indeed, the properties of these SP resonances, i.e. their strength and energy position, are extremely sensitive to the morphology, the size or the shape of the nanoparticles. In this respect, the design of specific shapes and morphologies for these particles has been at the focus of an intense effort, using either wet chemical synthesis routes or lithography. ${ }^{2,8-10}$ A non-exhaustive list of shapes and morphologies includes spheres, rods, triangles, cubes, pyramids, core-shell and star-like nanoparticles for example. $^{11}$

SPRs are also associated with enhanced local fields in the vicinity of the nanoparticles, which has sparked an interest in the nonlinear optical properties of these particles too. Nonlinear optical processes involving simultaneously several photons are not efficient but their crosssections, otherwise extremely small, can be enhanced by SPRs. ${ }^{12}$ Second harmonic generation (SHG), the process whereby two photons at a fundamental frequency are converted into one photon at the second frequency, has thus also received attention as this is one of the simplest nonlinear optical phenomena. This process is forbidden within the electric dipole approximation in materials possessing a center of inversion. ${ }^{13}$ This approximation neglects the spatial dependence of the electromagnetic fields. Similarly, it is also forbidden for nanoparticles with a centrosymmetric shape within the same approximation, provided that these particles are 
much smaller than the wavelength of light. However, it has since been demonstrated that centrosymmetric shapes are only ideal shapes, and as such, never observed in real samples. The centrosymmetry rule associated with perfect shapes is consequently easily broken in real experiments. Besides, retardation of electromagnetic fields, effectively the dependence of the electromagnetic fields with the space coordinates, requires the incorporation of higher order multipoles, beyond the electric dipole approximation. ${ }^{14}$ This feature introduces a source of complexity in the full understanding of the SHG response from metallic nanoparticles and careful studies must be performed to identify the origin of the response as well as to disentangle the different contributions to the overall response. Based on an appropriate knowledge of this response, several schemes have been proposed to use the nonlinear optical properties of metallic nanoparticles with specifically designed morphologies for applications, such as nanorulers and nonlinear plasmonic sensors for instance. ${ }^{15-17}$

Fundamental studies on the nonlinear optical properties have been performed over the last years on a series of gold and silver nanoparticles with a special attention to the shape of the nanoparticles. Nanospheres, nanorods and nanodecahedra have been closely investigated with the determination of their first hyperpolarizability, namely their cross-section for the SHG process. ${ }^{18-23}$ A competition between the shape defect contribution and the field multipole modes has been identified while the nonlinearity can be assumed to originate from the surface itself. ${ }^{24}$ Unfortunately, for many nanoparticle fabrication routes, be it in a liquid solution by the reduction of metallic salts or on a substrate by lithography, no perfect centrosymmetric shape can be achieved and it is difficult to disentangle these different contributions. Recently, it has nevertheless been demonstrated how polarization resolved measurements could achieve this operation. ${ }^{14}$ The shape defect contribution and the field multipoles have clearly been observed for nanospheres, nanorods and nanopyramids. In the latter case, the centrosymmetry of the 
second harmonic sources is accidental, due to a symmetry five cancellation. ${ }^{22}$ To go further into this direction, nanocubes are investigated in the present work. It is indeed expected that, because the six cube facets can be easily identified, the nanocube shape will be closer to the ideal shape, perhaps to a larger extent as compared to nanospheres and nanorods. ${ }^{20,21}$ After an introductory part presenting the morphology and the linear optical properties of the different samples, the first hyperpolarizability is measured using the standard method of hyper Rayleigh scattering (HRS) also known as second harmonic scattering (SHS). In the second part, a polarization analysis is performed for the different samples to investigate the contributions to the quadratic nonlinear optical response. Finally, these data are compared to surface integral method computations with a particular attention given to the role of a rounding procedure on the edges and corners.

\section{MATERIAL AND METHODS}

Chemicals. Silver nitrate $\left(\mathrm{AgNO}_{3}, 99+\%\right)$, ethylene glycol (EG, 99.8\%), polyvinylpyrrolidone (PVP, $\left.\mathrm{M}_{\mathrm{W}} \approx 55,000\right)$, sodium sulfide, hydrochloric acid (38\%), ethanol $(95 \%)$ were all purchased from Sigma-Aldrich and used as obtained.

Synthesis: Three silver nanocube samples with average edge lengths of $39 \pm 5.5 \mathrm{~nm}$ (sample $\mathrm{S} 1), 48 \pm 4.4 \mathrm{~nm}$ (sample S2), and $100 \pm 9.0 \mathrm{~nm}$ (sample S3) were prepared using variations of a sulfide-mediated polyol process developed by Xia et al.. ${ }^{25}$ First, for Samples 1 and 2, $6 \mathrm{~mL}$ of EG was heated at $140^{\circ} \mathrm{C}$ for $1 \mathrm{~h}$ in a $24 \mathrm{~mL}$ loosely closed reaction vial. This solution was continuously stirred throughout the whole reaction. Towards the end of the initial heating period, separate EG solutions were prepared containing PVP $(20 \mathrm{mg} / \mathrm{mL}), \mathrm{AgNO}_{3}(48 \mathrm{mg} / \mathrm{mL})$ and $\mathrm{Na}_{2} \mathrm{~S}(3 \mathrm{mM})$. Next, $90 \mu \mathrm{L}$ of the $\mathrm{Na}_{2} \mathrm{~S}$ solution was added and after an additional $\sim 9$ mins this was followed with $1.5 \mathrm{~mL}$ of the PVP solution. Then $0.5 \mathrm{~mL}$ of the $\mathrm{AgNO}_{3}$ solution was also 
added. After $\sim 15$ mins, when the reaction solution had turned to dark-green, the reaction was quenched by placing in a water bath at room temperature. Clean-up was performed by adding $\sim 50 \mathrm{~mL}$ of acetone and centrifuging at $2000 \mathrm{~g}$ for 30 mins. The supernatant was removed and the pellet of cubes was redispersed in distilled water. This was centrifuged again at $9000 \mathrm{~g}$ for 10 mins, the supernatant was discarded and the process was repeated a further two times. In the case of Sample 3, a modified procedure was adopted, ${ }^{26}$ where $5 \mathrm{~mL}$ of EG was heated for $1 \mathrm{~h}$, after which $1 \mathrm{~mL}$ of a freshly prepared solution of $3 \mathrm{mM} \mathrm{HCl}$ was added. Ten minutes later, EG solutions of $\mathrm{AgNO}_{3}(94 \mathrm{mM}, 3 \mathrm{~mL})$ and PVP $(147 \mathrm{mM}, 3 \mathrm{~mL})$ were added sequentially. The reaction was continued with heating at $140^{\circ} \mathrm{C}$ for $26 \mathrm{~h}$. Washing was performed by diluting in ethanol, centrifugation and resuspending the precipitate in water. This was repeated before filtering the aqueous solution through a nylon filter (Whatman, pore size: $0.45 \mu \mathrm{m}$ ).

\section{Scanning Electron Microscopy and UV-Vis Absorption Characterization}

The samples were characterized by scanning electron microscopy (SEM) to measure the nanocube edge length. Typical SEM pictures for the three samples are provided as Figures S1a$\mathrm{c}$ in the Supporting Information.

Similarly, the UV-visible extinction spectra were recorded for the three samples as well and are reported in Figure 1. 


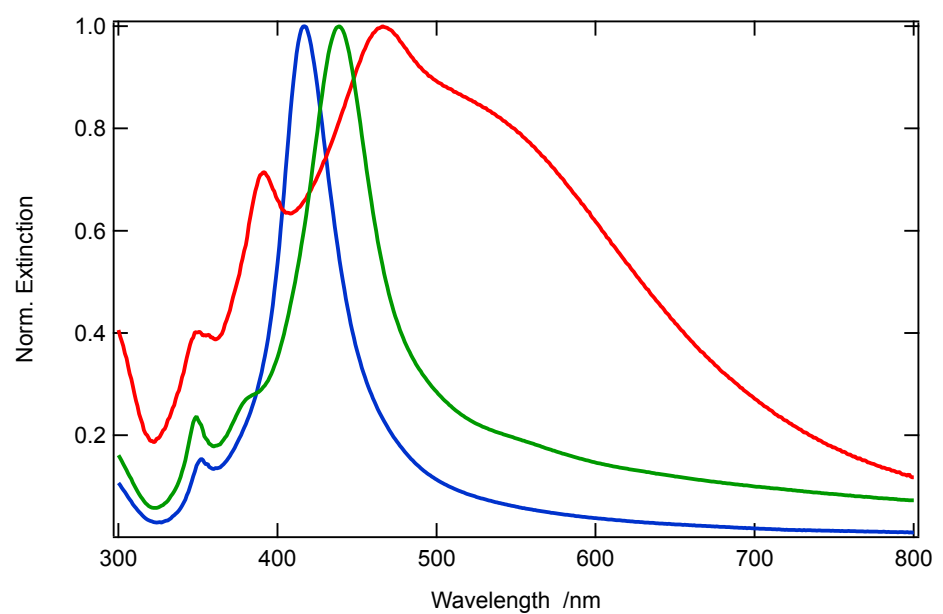

Figure 1 : Normalized UV-visible extinction spectra for the three silver nanocubes solution samples. (blue) Sample S1 : $39 \mathrm{~nm}$ edge length nanocubes, (green) Sample S2 : $48 \mathrm{~nm}$ edge length nanocubes and (red) Sample S3 : $100 \mathrm{~nm}$ edge length nanocubes.

The observed nanocube extinction spectra follow the trend already reported..$^{8,9,27}$ It is nevertheless pointed out that Sample S3 has a rather broad spectrum, indicative of a broad distribution of nanocubes size. All samples exhibit higher order multipolar resonances on the short wavelength side of the main electric dipole SP resonance.

\section{Nonlinear Optical Setup}

The experimental nonlinear scattering apparatus was based on a femtosecond laser source operating at a repetition rate of $80 \mathrm{MHz}$, a fundamental wavelength of $800 \mathrm{~nm}$ and delivering 140 femtosecond duration pulses with average power of about $700 \mathrm{~mW}$ at the laser exit. The fundamental beam linear polarization angle was selected with a half-wave plate and was spectrally cleaned with a low-pass filter to remove any harmonic light generated prior to the cell. The cell containing $1 \mathrm{~mL}$ of the nanocube aqueous samples was made of fused silica. The harmonic output light was then passed through a high-pass filter to remove the fundamental light and then sent at right angle from the fundamental beam propagation direction or in the forward direction through a collecting lens into a spectrometer coupled to a photomultiplier 
tube working in photon counting regime (see Figure S2). An analyzer was placed before the spectrometer to record the polarization plots.

\section{RESULTS AND DISCUSSION}

\section{First Hyperpolarizability}

The first hyperpolarizabilities of the silver nanocubes samples was determined by the standard technique of hyper Rayleigh scattering (HRS). The method offers the advantage of a measurement without any external symmetry-breaking element like a substrate. The quadratic nonlinear optical response stems from the nanocubes and the solvent and the latter is used as internal reference. The determination of the first hyperpolarizabilities entails the measurement of the harmonic scattered intensity as a function of the nanocubes concentration. The HRS intensity $I_{H R S}$ is then written as: ${ }^{28,29}$

$$
I_{H R S}=G\left(N_{S}\left\langle\beta_{S}^{2}\right\rangle+N_{n C}\left\langle\beta_{n C}^{2}\right\rangle\right) I^{2}
$$

where $G$ is a general constant and $I$ is the fundamental beam intensity. Also, $N_{S}$ and $N_{n C}$ are the number of water molecules, i.e. the solvent, and nanocubes per unit volume and $\beta_{S}$ and $\beta_{n C}$ their respective first hyperpolarizabilities. The brackets indicate an averaging procedure over all orientations in space since the measurements are performed in an isotropic liquid suspension. Figure 2 displays the data obtained for the three samples and Table 1 the first hyperpolarizabilities determined using the known first hyperpolarizability for pure water, namely $\beta_{S}=0.087 \times 10^{-30}$ esu..$^{30}$ The corresponding values measured previously for the first hyperpolarizability of silver nanospheres with different diameters are also given for comparison. 


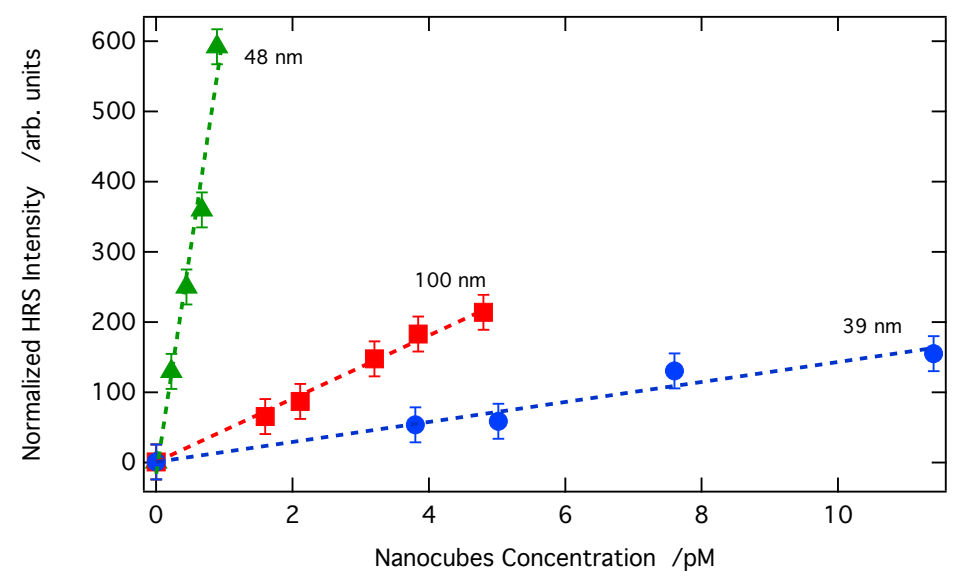

Figure 2 : $\quad 800 \mathrm{~nm}$ excited HRS intensity normalized to that of the neat water solution as a function of the concentration of (disks) $39 \mathrm{~nm}$, (triangles) $48 \mathrm{~nm}$ and (squares) $100 \mathrm{~nm}$ edge length nanocubes.

Table 1 : $\quad$ First Hyperpolarizability $\beta$, first hyperpolarizability corrected for resonance enhancement $\beta_{0}$ and first hyperpolarizability corrected for resonance enhancement and divided by the number of silver atoms in the particle measured at $800 \mathrm{~nm}$ fundamental wavelength for the three nanocubes samples. Similar values provided for silver nanospheres for comparison (taken from Ref. 31). The nanocube equivalent diameter $D$ is the diameter of a spherical nanoparticle with same volume.

\begin{tabular}{cccccc}
\hline Sample & $\begin{array}{c}\text { Edge } \\
\text { Length } \\
n m\end{array}$ & $\begin{array}{c}\times \mathbf{1 0}^{\mathbf{2 5}} \boldsymbol{\beta} \\
\text { esu }\end{array}$ & $\begin{array}{c}\boldsymbol{D} \\
n m\end{array}$ & $\begin{array}{c}\times \mathbf{1 0}^{\mathbf{2 5}} \boldsymbol{\beta}_{\mathbf{0}} \\
\text { esu }\end{array}$ & $\begin{array}{c}\times \mathbf{1 0}^{\mathbf{3 2}} \overline{\boldsymbol{\beta}_{\mathbf{0}}} \\
\text { esu/atom }\end{array}$ \\
\hline S1 & 39 & $10.6 \pm 1.1$ & 33.5 & $0.5 \pm 0.05$ & $1.4 \pm 0.2$ \\
S2 & 48 & $16.6 \pm 1.7$ & 41.3 & $1.8 \pm 0.2$ & $2.7 \pm 0.3$ \\
S3 & 100 & $12.8 \pm 1.3$ & 86.0 & $3.4 \pm 0.3$ & $0.6 \pm 0.1$ \\
Nanospheres & - & $7.0 \pm 0.7$ & 40 & $0.3 \pm 0.1$ & $0.5 \pm 0.1$ \\
Nanospheres & - & $30.1 \pm 3$ & 80 & $5.3 \pm 0.5$ & $1.4 \pm 0.2$ \\
\hline
\end{tabular}

The silver nanocubes' first hyperpolarizability present a behavior with the largest magnitude for the $48 \mathrm{~nm}$ edge length nanocubes for a $800 \mathrm{~nm}$ excitation wavelength. This behavior must be analyzed in light of the corresponding absorption spectra. The SPR are indeed rather sharp for nanocubes and therefore the field enhancement spectrally narrow.

To rationalize the first hyperpolarizabilities, it is appropriate to correct them for the number of atoms in the nanoparticles and for the SPR field enhancement. This scaling with the number of atoms corresponds to a volume scaling, as expected when field retardation is taken 
into account. A surface scaling of the first hyperpolarizability could have also been used because silver metal is a centrosymmetric medium at the atomic level. However, because retardation plays a non-negligible role and introduces orders beyond the electric dipole one, such a scaling is not appropriate. Besides, this analysis is further supported considering the centrosymmetry of the nanocube shape. Indeed, within the local electric dipole approximation, the first hyperpolarizability vanishes altogether leaving a response dominated by higher order field multipoles, i.e. retardation. The rescaling of the first hyperpolarizabilities determined experimentally is performed in Table 1 . The second correction introduced is required by the SP resonance field enhancement. As observed on the extinction spectra (see Figure 1), there is a spectral shift of the maximum of the extinction spectrum as a function of the nanocubes edge length. As a result, the measured hyperpolarizabilities are resonantly enhanced by the SP resonance excitation. In the present case, the enhancement can be considered to occur essentially at the second harmonic wavelength, namely $400 \mathrm{~nm}$. In order to correct for this enhancement, the two level model is applied. ${ }^{32}$ No analytical expression is available for the field factors of the second harmonic response from nanocubes, to the contrary of nanospheres or nanoellipsoids. The two level model correction procedure used here is customary in molecular organic nonlinear optics to compare static hyperpolarizabilities when resonance enhancement occurs at different wavelengths. ${ }^{33}$ Hence, Table 1 displays the first hyperpolarizabilities corrected for the resonance enhancement using the resonance wavelengths determined from the UV-visible absorption spectra. The resonance enhancement factor $f(\omega)$ in the two level model is simply given by $\beta=\beta_{0} f(\omega)$ with :

$$
f(\omega)=\frac{\omega_{0}^{4}}{\left(\omega_{0}^{2}-\omega^{2}\right)\left(\omega_{0}^{2}-4 \omega^{2}\right)}
$$


where $\omega_{0}$ is the resonance frequency and $\omega$ the excitation fundamental frequency. All of the hyperpolarizabilities after correction for resonance enhancement and rescaled by the number of silver atoms contained on average in the nanocubes, present a magnitude close to $\sim 1 \times 10^{-32}$ esu/atom, leading to a generic value for long edge length silver nanocubes. This value compares rather well with the one obtained for spherical silver nanoparticles, also shown in Table 1. Consequently, when field retardation is non-negligible due to the nanoparticles size, the shape does not play a leading role anymore, at least for the sub-class of nanoparticles with a centrosymmetric shape.

\section{Polarization Analysis}

The nanocubes edge length investigated range from 39 to $100 \mathrm{~nm}$, a range where field retardation, namely the dependence of the electromagnetic fields with the spatial coordinates of the nanoparticles, is known to play a major role in the response in the case of nanospheres. ${ }^{14}$ The role of retardation is also confirmed for nanocubes using polarization analysis of the HRS intensity. To investigate this point further, HRS studies were performed where the input angle of polarization $\gamma$ of the linearly polarized fundamental beam was rotated for fixed output polarization angles. This method has been demonstrated in the past to be ideally suited to identify the contributions of the electric quadrupole and octupole for the second harmonic scattering from nanospheres. In Figure 3 are thus given the different $\gamma \mathrm{V}$ and $\gamma \mathrm{H}$ plots, where $\mathrm{V}$ and $\mathrm{H}$ stands for vertically and horizontally polarized second harmonic light, obtained for the different samples. The V polarized plots clearly show a four lobes pattern for all edge lengths. This pattern is a typical signature of retardation in nano-objects and more particularly of the second harmonic quadrupole mode. The weight of this contribution increases with the edge length and is almost fully developed for the $100 \mathrm{~nm}$ edge length nanocubes, as seen from the quantitative analysis performed below. 
(a)

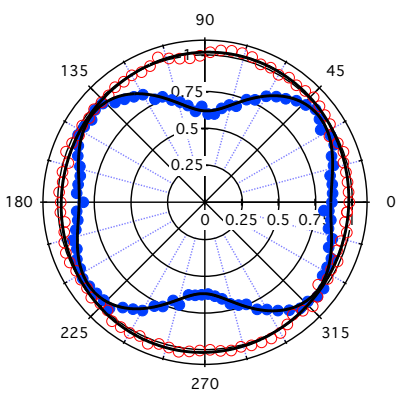

(b)

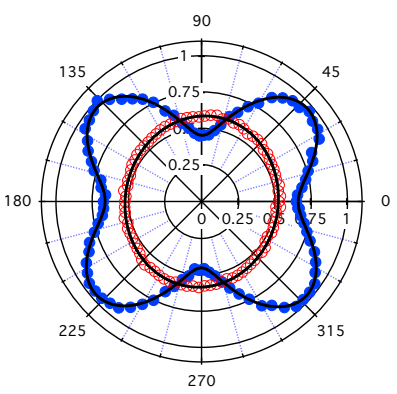

(c)

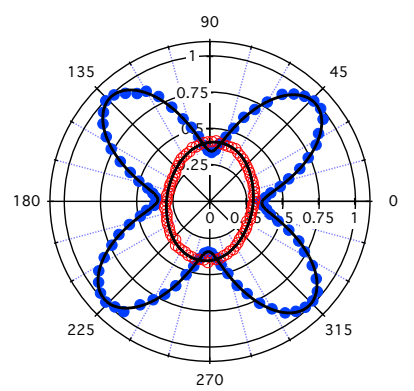

Figure 3 : $\quad$ Right angle polarization plots of the HRS intensity excited at $800 \mathrm{~nm}$ for the (a) $39 \mathrm{~nm}$, (b) $48 \mathrm{~nm}$ and (c) $100 \mathrm{~nm}$ edge length nanocubes : (full blue disks) V polarized harmonic intensity, (empty red disks) $\mathrm{H}$ polarized harmonic intensity.

On the contrary, the $\mathrm{H}$ polarized plots exhibit weakly varying intensities as a function of the angle $\gamma$. At a $39 \mathrm{~nm}$ edge length, the plot is a circle and it evolves into an ellipse at longer edge lengths. This feature indicates a contribution from retardation attributed to the second harmonic octupolar mode, similarly to the case of centrosymmetric nanospheres with large diameters. ${ }^{14}$ A quantitative analysis can be built with four parameters to describe the polarization dependent results. The first one, $\zeta_{V}$, defines the relative weight of the excited second harmonic quadrupolar mode against the surface defect contribution. As said above, the latter contribution arises from the deviation of the shape of the nanocubes from the perfect cubic shape. We hence define: ${ }^{18}$

$$
\zeta_{V}=\frac{I_{45 V}-\left[I_{\mathrm{vV}}+I_{h V}\right]}{I_{45 V}}
$$

where $I_{45 V}, I_{\mathrm{v} V}$ and $I_{h V}$ are the HRS intensities collected for the input polarization $\gamma$ equal to $45^{\circ}, 0^{\circ}(\mathrm{v})$ and $90^{\circ}(\mathrm{h})$ respectively and the harmonic intensity vertically $\left(0^{\circ}\right.$ or $\left.\mathrm{V}\right)$ polarized. The non-vanishing $I_{\mathrm{v} V}$ and $I_{h V}$ intensities experimentally measured assess the imperfect nanocubes shape. Indeed, for perfect nanocubes or centrosymmetric shape, the nanocubes should exhibit vanishing $I_{\mathrm{v} V}$ and $I_{h V}$ intensities. The experimental values of $\zeta_{V}$ are given in 
Table 2 for the as-synthesized nanocubes with respective edge lengths of 39, 48 and $100 \mathrm{~nm}$. These values all differ from unity, indicating imperfect cubic shapes. Their increase with the nanocube edge length also indicates that the contribution from the excited second harmonic quadrupole increases with the nanocubes edge length as compared to the shape defect contribution. The non vanishing $I_{\mathrm{v} V}$ and $I_{h V}$ intensities observed can tentatively be attributed to the role of the corners and edges, highly confined regions where it is expected that the centrosymmetry rule can be broken quite easily, see below for an in-depth discussion about the corners contribution. In a similar way, for the $\mathrm{H}$ polarized plots, the parameter $\zeta_{H}$ can be calculated as: ${ }^{14}$

$$
\zeta_{H}=\frac{I_{\max }-I_{\min }}{I_{\max }+I_{\min }}
$$

where $I_{\max }=\max \left(I_{\mathrm{v} H}, I_{h H}\right)$ and $I_{\min }=\min \left(I_{\mathrm{v} H}, I_{h H}\right)$. This parameter permits to quantify the second harmonic octupolar mode contribution. The $\zeta_{H}$ value increases with the edge length, from a vanishing value indicative of the absence of the octupole contribution to a finite value in agreement with an increasing contribution from retardation, i.e. an increasing octupolar contribution.

\section{Unequal Contributions from the Cube Corners}

To get a deeper insight into the origin of the nanocubes HRS response, it is possible to record the second harmonic scattered in the forward direction. In this case, the multipolar contributions associated with field retardation are suppressed. 
Table 2 : $\quad$ Parameters determined for the different nanocubes samples polarization plots. See text for the definitions of the four parameters.

\begin{tabular}{cccccc}
\hline Sample & $\begin{array}{c}\text { Edge } \\
\text { Length } \\
n m\end{array}$ & $\xi_{V}$ & $\zeta_{H}$ & $D_{90}$ & $D_{180}$ \\
& 39 & $0.37 \pm 0.11$ & $0.02 \pm 0.05$ & $0.73 \pm 0.25$ & $0.64 \pm 0.21$ \\
S1 & 48 & $0.59 \pm 0.2$ & $0.06 \pm 0.05$ & $0.69 \pm 0.24$ & $0.51 \pm 0.15$ \\
S2 & 100 & $0.78 \pm 0.25$ & $0.16 \pm 0.03$ & $0.97 \pm 0.32$ & $0.55 \pm 0.17$ \\
S3 & & & &
\end{tabular}

Then, repeating the same operation as before where the HRS intensity is collected for both crossed vertical and horizontal polarization states as a function of the input polarization angle $\gamma$. Two crossed dipolar patterns are recorded as seen on Figure 4 for the $48 \mathrm{~nm}$ edge length nanocubes resulting from the symmetry of the experiments itself. The corresponding graphs for the 39 and $100 \mathrm{~nm}$ edge lengths are similar and are provided in Figure S3 in the Supplementary Information.

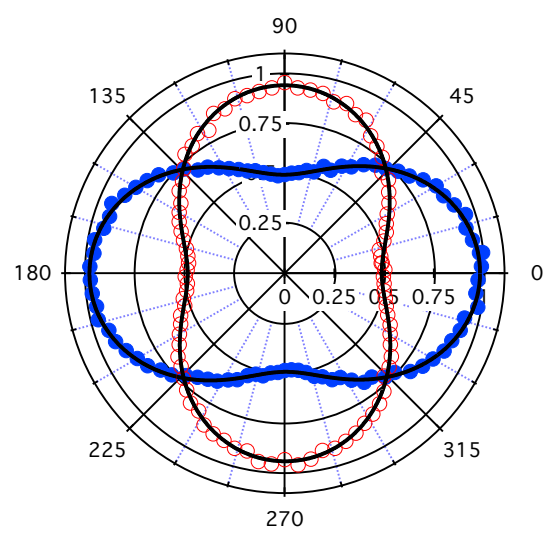

Figure 4: $\quad$ Forward polarization plots of the HRS intensity excited at $800 \mathrm{~nm}$ for the $48 \mathrm{~nm}$ edge length nanocubes: (filled blue disks) V polarized harmonic intensity, (empty red disks) H polarized harmonic intensity.

For perfectly centrosymmetric nanoparticles, no signal should be recorded in this configuration. Indeed, only the occurrence of deviations from centrosymmetry introduces a non-vanishing surface defect contribution that can be observed in this geometrical forward 
configuration without the contribution from field retardation. Hence, Figure 4 clearly exhibits the unbalanced contributions of the eight cube corners as these corners can be indeed assumed to be the sources of the second harmonic light from plasmonic nanocubes.

In a more quantitative way, a third parameter and a fourth parameter are worth discussing, namely $D_{90}=I_{h V} / I_{\mathrm{v} V}$ recorded with the collection direction at $90^{\circ}$ from the fundamental beam propagation and likewise $D_{180}=I_{h V} / I_{\mathrm{v} V}$ collected for the forward direction at $180^{\circ}$. The two ratios are rather similar and take values between 0.5 and unity. The latter ratios are related to the nanocubes 3D nonlinear tensorial response stemming from the cubes corners. Similarly to molecular systems, this ratio provides a further insight into the tensorial dipolar and octupolar weight to the first hyperpolarizability. Indeed, an irreducible decomposition of the first hyperpolarizability tensor yields $\vec{\beta}=\vec{\beta}^{(1)} \oplus \vec{\beta}^{(3)}$ where $\vec{\beta}^{(1)}$ and $\vec{\beta}^{(3)}$ are respectively the dipolar and octupolar irreducible hyperpolarizability tensors. ${ }^{34,35}$ Note here, that this decomposition must not be mistaken with the field multipoles discussed above and arising from retardation. For 3D systems and without making any further hypotheses on the first hyperpolarizability tensor elements, the problem is rather complicated. It nevertheless sheds light on the symmetry of this response associated with the perfectness of the nanocubes shape. Considering the values of the ratio $D_{90}$ and $D_{180}$ observed, all between 0.5 and unity, see Table 2, it can be concluded that the deviation of the perfect cubic shape yields a first hyperpolarizability dominated by a tensorial octupolar symmetry.

\section{Simulation of Dipolar and Octupolar Tensorial Nanocube Hyperpolarizabilities}

In order to further support the above analysis of the response from the nanocubes, a model was developed. A first hyperpolarizability was attributed to each corner and distributed in space according to the eight nanocube corners location. To simplify the problem, each corner 
hyperpolarizabiltiy was assumed to have a single non vanishing tensor element $\beta_{z z z}$ where the corner $O z$ direction is oriented outward along the direction going from the center of the nanocube and out through the corner. Then, all averaging procedures were performed to account for the HRS experiment in a liquid solution. Two distributions of the corners first hyperpolarizabilities were investigated as described in Figure 5. On this Figure, the color code indicates the hyperpolarizability tensor element $\beta_{z z z}$ value. The filled versus empty red disk code indicates values differing by $\pm \Delta \beta_{z z z}$ respectively with $\pm \Delta \beta_{z z z} / \beta_{z z z}=0.1$ whereas as the blue disk code indicates unit tensor elements. Note that all simulations can be performed in relative units, i.e. with a unit $\beta_{z z z}$ tensor element, since one is interested in the intensity normalized $D_{90}$ and $D_{180}$ ratios. One distribution is therefore dipolar, oriented along one of the nanocube diagonals whereas the other one is octupolar in the irreducible tensorial decomposition sense. These two simple examples constitute only a small subset of the possibilities afforded by the eight corners nanocube geometry. The number of the possible configurations is indeed very large, especially when incorporating other tensor elements for each corner hyperpolarizability to account for the local symmetry. This could be the case for instance in order to account for the neighboring edges. 


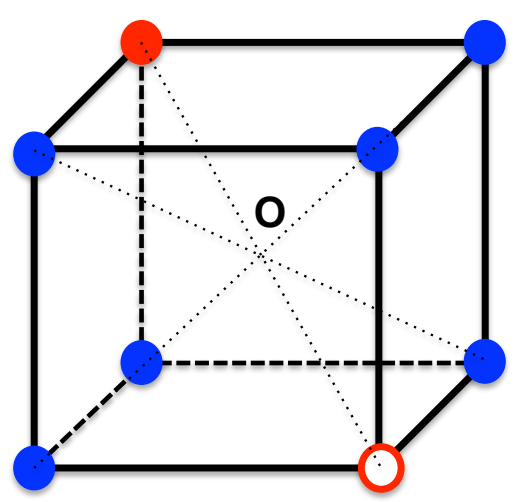

(a) dipolar

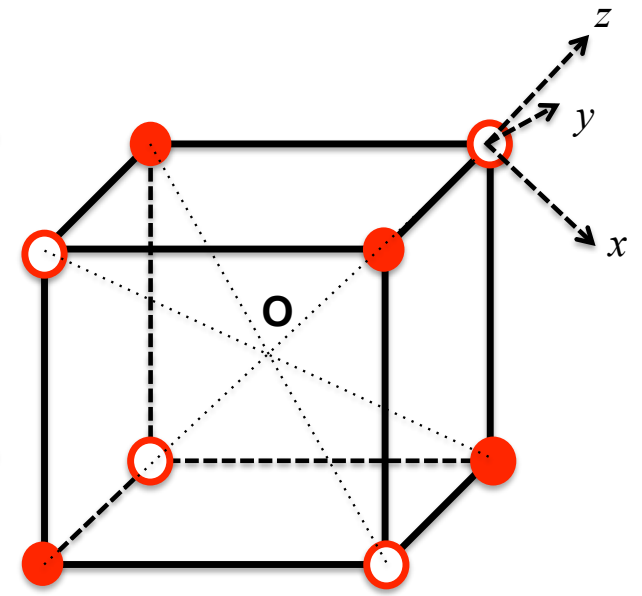

(b) octupolar

Figure 5(a-b) : Two distributions of the eight corners first hyperpolarizability tensor investigated : (a) dipolar distribution, (b) octupolar distribution. Blue disks describe corners associated with a unit first hyperpolarizability $\beta_{z z z}$, filled red disks with corners associated with a first hyperpolarizability equal to $\beta_{z z z}+\Delta \beta_{z z z}$, and empty red disks with a first hyperpolarizability equal to $\beta_{z z z}-\Delta \beta_{z z z}$ where $\Delta \beta_{z z z}=0.1$. Hyperpolarizability units are arbitrary. A local corner reference frame is given as an example for a corner of the octupolar configuration.

The simulations were performed for both the right angle and the forward geometry with the following results reported in Figure 6 for two edge lengths, namely a short $5 \mathrm{~nm}$ one where retardation is minimal and a longer edge length of $80 \mathrm{~nm}$ where retardation dominates. It is important to note that the sizes introduced in the simulation do not match perfectly the experimental ones because of the interplay between the first hyperpolarizability values and sizes that is difficult to quantitatively account for. From a symmetry point of view, the dipolar distribution is unlikely to occur. It is nevertheless displayed as Figure S4 in the Supplementary Information file. The corresponding polar graphs for the octupolar distribution describe the experimental data with excellent agreement. 


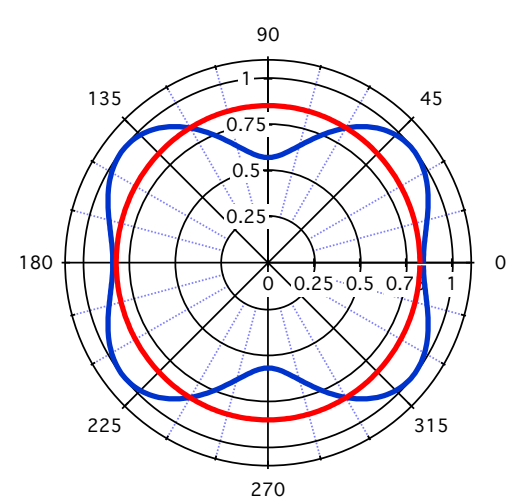

(a)

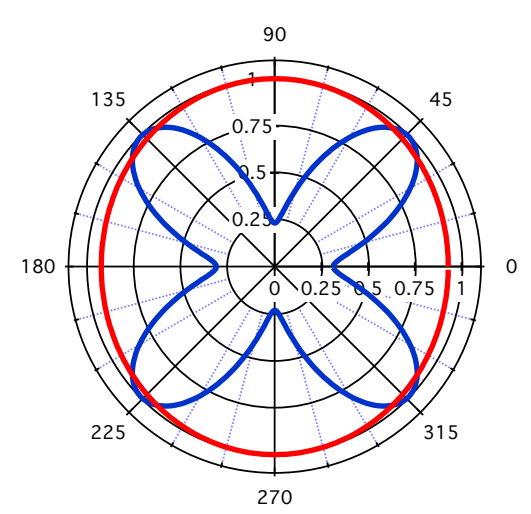

(c)

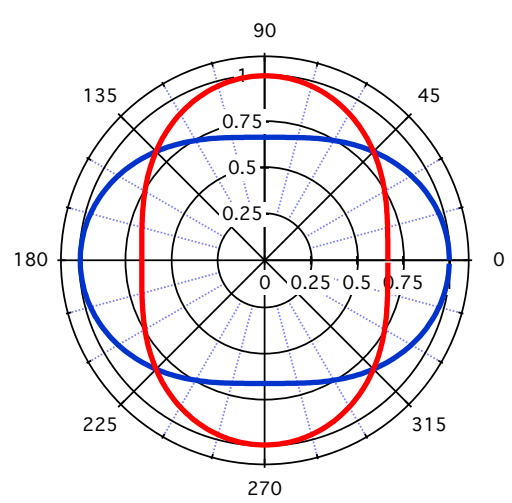

(b)

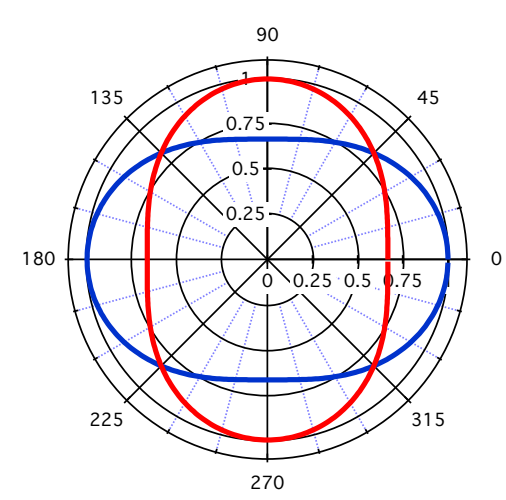

(d)

Figure 6(a-d) : $\quad$ Simulated polarization resolved HRS plots for two different sizes of the octupolar distribution of the corners hyperpolarizability. (Blue) Vertically polarized harmonic intensity, (red) Horizontally polarized harmonic intensity. (a) $5 \mathrm{~nm}$ edge length, right angle configuration, (b) $5 \mathrm{~nm}$ edge length, forward configuration, (c) $80 \mathrm{~nm}$ edge length, right angle configuration, (d) $80 \mathrm{~nm}$ edge length, forward configuration.

The simulated polarization resolved HRS plots for the octupolar distribution of the individual response of the corners first hyperpolarizability are, from a close comparison between the experimental and simulated $D_{90}$ and $D_{180}$ parameters reported in Tables 2 and 3 , in better agreement with the experimental data as compared to the polar distribution. This result further supports the analysis of the experimental parameters reported in Table 2 . The corresponding parameters of the simulated plots are reported in Table 3. 
Table 3 : Various parameters determined for the simulated nanocubes polarization plots. See text for the definitions of the parameters.

\begin{tabular}{ccccc}
\hline $\begin{array}{c}\text { Edge } \\
\text { Length } \\
n m\end{array}$ & $\zeta_{V}$ & $\zeta_{H}$ & $D_{90}$ & $D_{180}$ \\
\hline $\begin{array}{c}\text { Polar } \\
\mathbf{5}\end{array}$ & 0.30 & 0.03 & 0.30 & 0.2 \\
$\mathbf{8 0}$ & 0.84 & 0.04 & 0.74 & 0.2 \\
Non Polar & & & & \\
$\mathbf{5}$ & 0.44 & 0.01 & 0.68 & 0.67 \\
$\mathbf{8 0}$ & 0.84 & 0.04 & 0.75 & 0.67 \\
\hline
\end{tabular}

\section{Surface Integral Equation Calculations}

To obtain another insight into the nonlinear optical properties of silver nanocubes, the second harmonic response was numerically evaluated using a surface integral equation (SIE) method. This method has been extensively described elsewhere. ${ }^{36}$ The method requires in particular the discretization of the scattering object surface only and is thus suitable for the indepth investigation of rounding and size effects in silver nanocubes. The dielectric constants for silver were taken from Johnson and Christy at both the fundamental and second harmonic wavelengths. ${ }^{37}$ All the computations were performed considering nanocubes dispersed in water with optical index $n=1.33$. The SHG response was computed taken into account a surface contribution only for the nonlinear optical response. It confirms in particular that the nanocubes nonlinear optical response arises from the corners, see Figure 7 below.
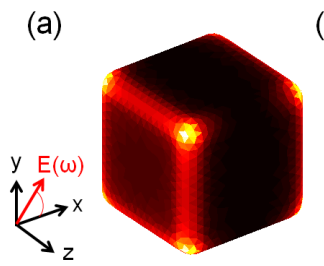

(b)

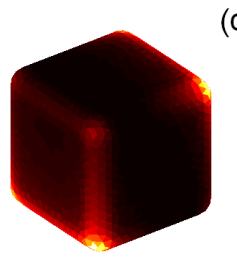

(c)

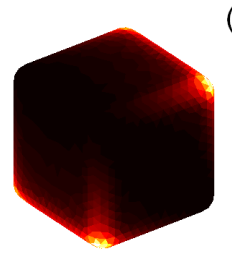

(d)

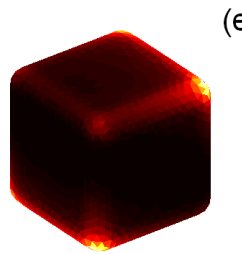

(e)

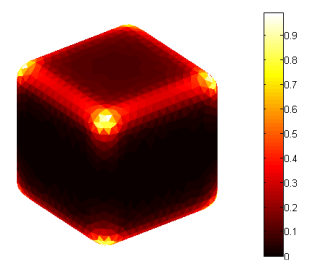

Figure 7 : $\quad$ Surface second harmonic polarization excited at $800 \mathrm{~nm}$ for a $40 \mathrm{~nm}$ silver nanocube with an edge and corner rounding of $5 \mathrm{~nm}$ for an incident polarization angle (a) $\gamma=0^{\circ}$, (b) $\gamma=22.5^{\circ}$, (c) $\gamma=45^{\circ}$, (d) $\gamma=67.5^{\circ}$, and (e) $\gamma=90^{\circ}$. 
The impact of the nanocube size is investigated first. The scattering spectra of the silver nanocubes with various sizes, edge length $a$ ranging from $40 \mathrm{~nm}$ to $100 \mathrm{~nm}$, correspond to the range of nanocube edge lengths experimentally investigated and are shown in Figure 8a. The edges and corners were rounded with a rounding radius $r=a / 4$ following the procedure described in the inset of Figure 8a. All spectra exhibit several resonances, similarly to the experiments as seen in Figure 1. For each spectrum, the strongest resonance corresponds to the excitation of the dipolar SP resonance. As the nanocube size increases, this resonance redshifts and broadens due to an increase in radiative losses.

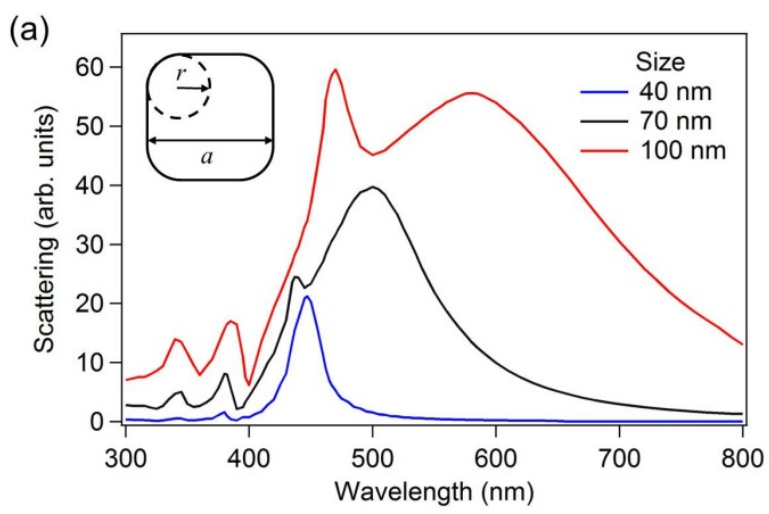

(b)

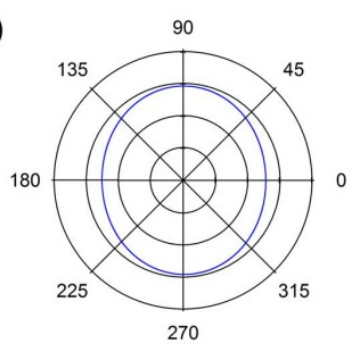

(d)

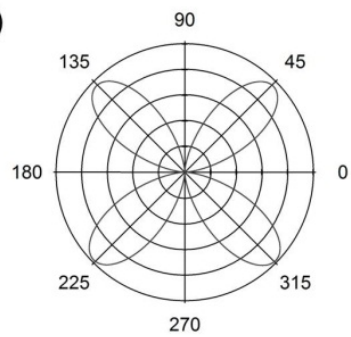

(c)

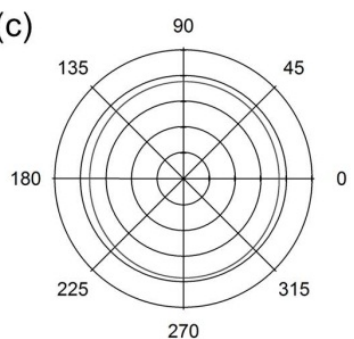

(e)

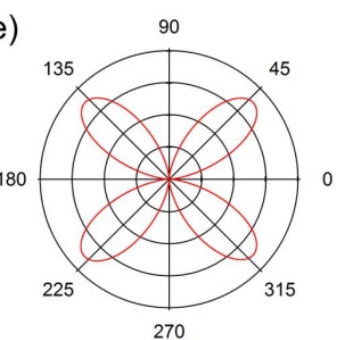

Figure 8(a-e): (a) Second harmonic scattering cross-section as a function of the incident wavelength computed for silver nanocubes with edge length $a=40 \mathrm{~nm}$ (blue), $70 \mathrm{~nm}$ (black) and $100 \mathrm{~nm}$ (red). The edges and corners were rounded with a rounding radius $r=a / 4$ (see inset). Relative second harmonic intensity scattered in the vertical plane as a function of the scattering direction evaluated for (b-c) a $\mathrm{H}$ polarized second harmonic wave (polarized along the incident wave 
propagation vector) and (d-e) a $\mathrm{V}$ polarized second harmonic wave. The edge length $a$ is (b) $40 \mathrm{~nm},(\mathrm{c}-\mathrm{d}) 70 \mathrm{~nm}$ and (e) $100 \mathrm{~nm}$.

In the nonlinear regime, the relative second harmonic intensity scattered in the vertical plane as a function of the scattering direction were evaluated for a second harmonic wave polarized along the incident wave (see Figures $8 b, c$ ). This configuration is closely related to HRS measurements where the SH wave is collected at right angle as presented in the above section albeit without any orientation distribution averaging. Note that the second harmonic intensity vanishes in the backward and forward directions for perfectly symmetric nanocubes like those numerically studied here. The fundamental wavelength was set to $800 \mathrm{~nm}$ in order to make comparisons with the experimental results straightforward. The case of the $\mathrm{H}$ polarized second harmonic wave (polarized along the incident wave vector) is discussed first. For the smallest nanocubes, i.e. $a=40 \mathrm{~nm}$, the second harmonic intensity slightly oscillates as the observation direction is rotated. This feature arises from interferences between the second harmonic excited dipolar and octupolar modes. These interferences are destructive or constructive depending on the scattering direction, as previously reported for the SHG response from metallic nanospheres. ${ }^{14}$ Note that the second harmonic dipole moment points along the propagating direction of the incident plane wave, contrary to the linear dipole moment which points along the incident electric field direction. For larger nanocubes, i.e. $a=70 \mathrm{~nm}$, the second harmonic intensity is found almost independent of the scattering direction for the $\mathrm{H}$ polarized second harmonic wave. Despite larger retardation effects, the second harmonic excited octupolar contribution is weaker since the first octupolar resonance is shifted to longer wavelengths, namely $440 \mathrm{~nm}$, see Figure 8a, resulting in a smaller interference contrast. This feature is however not so well observed experimentally. This discrepancy may arise from the dispersion in morphology of the nanocubes and the corresponding SP resonance band broadening. The relative second harmonic intensity scattered in the vertical plane as a function 
of the scattering direction was also evaluated for a $\mathrm{V}$ polarized second harmonic wave (see Figure $8 \mathrm{~d}, \mathrm{e})$. In this case, a four-lobe emission pattern corresponding to a second harmonic quadrupolar mode is observed, similarly to the experimental results presented above, see Figure 3. Even though the emission patterns are identical, the lobe intensity is about 70 times higher for a $100 \mathrm{~nm}$ nanocube than for a $70 \mathrm{~nm}$ nanocube. Experimentally, this edge length range corresponds to a regime where the scattered intensity scales with the volume of the nanocube. Experimentally therefore, a factor of three only is expected. Thus, this large change can be understood as arising from the redshift of the dipolar resonance as the nanocube size increases, see Figure 1. Indeed, it has already been shown that a dipolar resonance excitation at the fundamental wavelength is preferred for the excitation of the second harmonic excited quadrupolar mode. These calculations are in agreement with the experimental data discussed above. In order to go forward with the investigation of the impact of the nanocube shape on the SHG, the effect of the corners and edges rounding is now considered in greater detail.

The impact of the corners' and edges' rounding procedure on the linear response of silver nanocubes was recently discussed and it was shown that an increase of the rounding radius $r$ blueshifts the dipolar SP resonance towards the SP resonance supported by a sphere with the same size. ${ }^{38}$ To quantify this rounding effect and perform a comparison between nanocubes with different edge lengths, a rounding parameter $R$ defined as $R=2 r / a$ is introduced. The parameter $R$ evolves from 0 for cubes with sharp corners to 1 for a perfect nanosphere (see Figure 9a). The relative second harmonic intensity scattered in the vertical plane with a scattering angle equal to $0^{\circ}$ evaluated for a $\mathrm{H}$ polarized second harmonic wave (polarized along the incident beam) is then shown as a function of the rounding parameter $R$ for a total edge length $a=40 \mathrm{~nm}$ and $70 \mathrm{~nm} .{ }^{39}$ As the rounding parameter $\mathrm{R}$ increases, i.e. as the nanoparticles evolves from nanocubes with sharp corners to nanospheres, the second harmonic intensity 
increases for the two considered nanocube sizes. This observation is explained by the blueshift of the dipolar SP resonance. As the corners rounding increases, this resonance shifts closer to the $400 \mathrm{~nm}$ emission wavelength resulting in an increase of the second harmonic excited dipolar mode contribution. The SP resonance wavelengths for the final spheres were estimated to be $398 \mathrm{~nm}(a=40 \mathrm{~nm})$ and $430 \mathrm{~nm}(a=70 \mathrm{~nm})$ respectively using Mie theory. The SPR wavelength for the $40 \mathrm{~nm}$ sphere is shorter than the SH wavelength explaining the slight second harmonic intensity decrease observed for rounding parameter $R$ close to unity (see Figure $9 \mathrm{~b}$ ). Let us now turn our attention to the effect of rounding corners on the $\mathrm{SH}$ excited quadrupolar emission. The relative second harmonic intensity scattered in the vertical plane, i.e. scattering angle equals to $45^{\circ}$ (see Figure $8 \mathrm{~d}, \mathrm{e}$ ), evaluated for a $\mathrm{V}$ polarized second harmonic wave (polarized in the vertical plane) is shown as a function of the rounding parameter for an edge length of $a=70 \mathrm{~nm}$ and $100 \mathrm{~nm}$ (see Figure 9c). In both cases, the SH intensity decreases as the rounding parameter $R$ increases. This behavior is also explained by a blueshift of the dipolar SP resonance. As mentioned above, a dipolar resonant excitation at the fundamental wavelength favors the $\mathrm{SH}$ quadrupolar mode but the dipolar SP resonance is shifted away from the fundamental wavelength at $800 \mathrm{~nm}$ as the rounding parameter increases. These results emphasize that the second harmonic response of plasmonic nanocubes is more complex than its linear counterpart due to the interplay between resonance effects at the fundamental and SH wavelengths. 
(a)
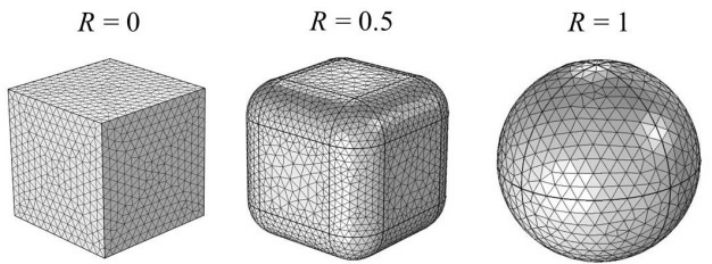

(b)

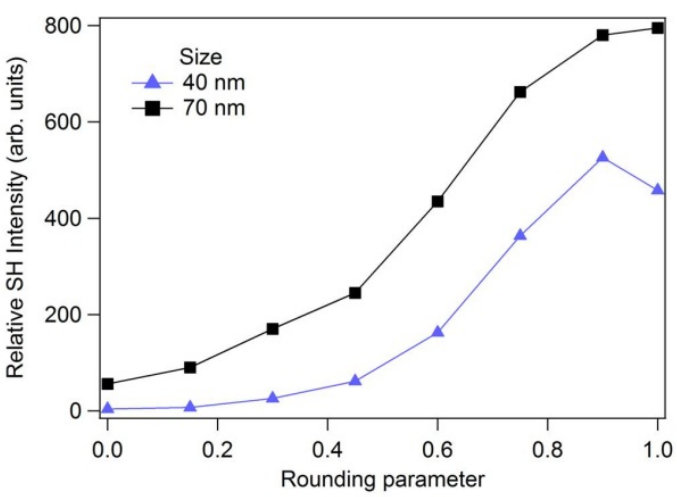

(c)

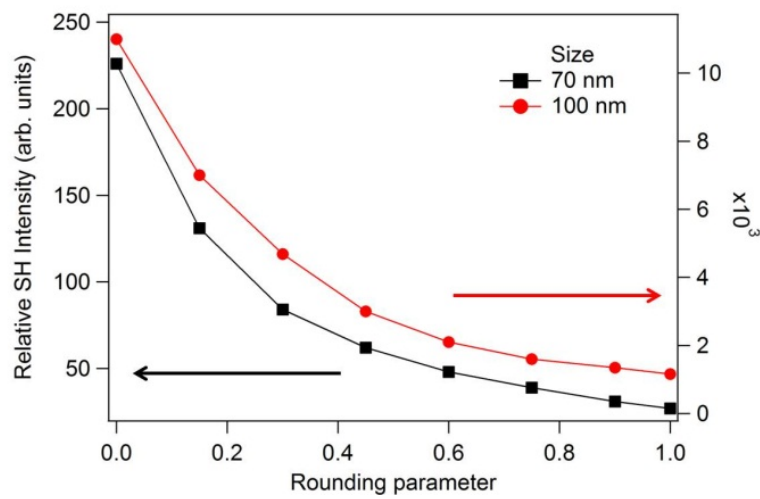

Figure 9(a-c): (a) Example of nanocubes for different values of the rounding parameter $R$. This parameter evolves from 0 (nanocube with sharp corners) to 1 (perfect nanosphere). (b) Relative SH intensity scattered in the vertical plane (scattering angle equals to $0^{\circ}$, see Fig. 5 (b-c)) evaluated for a $\mathrm{H}$ polarized second harmonic wave (polarized along the incident beam) as a function of the rounding parameter $R$ for an edge length $a$ of $40 \mathrm{~nm}$ (blue) and $70 \mathrm{~nm}$ (black). (c) Relative second harmonic intensity scattered in the vertical plane (scattering angle equals to $45^{\circ}$, see Fig. 5 (d-e)) evaluated for a V polarized second harmonic wave (polarized in the vertical plane) as a function of the rounding parameter for an edge length $a$ of $70 \mathrm{~nm}$ (black) and $100 \mathrm{~nm}$ (red).

\section{CONCLUSIONS}

The first hyperpolarizability for silver nanocubes has been determined for edge lengths between 39 and $100 \mathrm{~nm}$. It appears that in all cases the response can be described by a surface nonlinearity only with a strong contribution from field retardation. A value of the first 
hyperpolarizability per atom is deduced, corrected for the SP resonance enhancement, and found similar to the one obtained for centrosymmetric silver nanospheres. Also, a polarization analysis of the scattered second harmonic intensity scattered is performed, further supporting the hyperpolarizability analysis. The retardation contribution in the overall SHG response is clearly observed using the adequate polarization schemes. In the forward geometry, it is nevertheless possible to discuss the unbalanced role of the eight nanocube corners. Finally, further in depth analysis is brought in with surface integral method analysis to investigate the role of SP resonance enhancement as well as edge and corners rounding.

From this general analysis, it appears that nanocubes, although possessing a welldefined centrosymmetric shape, still retain a strong shape defect electric dipole contribution in their SHG, most likely due to the difficulty to establish a perfect centrosymmetry with edges and corners. This point is important for a future design of second order nonlinear plasmonic metasurfaces based on nanocubes.

\section{Supporting Information}

SEM images of the three nanocube samples, schematics of the experimental hyper Rayleigh set-up, simulation details, forward scattering polarization plots for the 39 and $100 \mathrm{~nm}$ edge length nanocubes, simulated scattering polarization plots for the dipolar distribution of the first hyperpolarizability nanocube corners, and geometry for the SIE computations.

\section{Acknowledgements}

IRA, ChJ, EB and PFB acknowledge the support from the Agence Nationale de la Recherche under contract ANR-17-CE24-0029 and Center for Nano-Optics Nanoptec of the University Claude Bernard Lyon 1. AWW thanks David Sleith for assistance with the nanocube synthesis. 


\section{References}

(1) Maier S.A. Plasmonics: Fundamentals and applications; Springer, New York, 2007

(2) Rycenga M.; Cobley C. M.; Zeng J.; Li W.; Moran C. H.; Zhang Q.; Qin D.; Xia Y. Controlling the Synthesis and Assembly of Silver Nanostructures for Plasmonic Applications. Chem. Rev. 2011, 111, 3669-3712

(3) McFarland A. D.; Van Duyne R. P. Single Silver Nanoparticles as Real-Time Optical Sensors with Zeptomole Sensitivity. Nano Lett. 2013, 3, 1057-1062

(4) Kawata S.; Inouye Y.; Verma P. Plasmonics for Near-Field Nano-Imaging and Superlensing. Nat. Photonics 2009, 3, 388-394

(5) Hess O.; Pendry J. B.; Maier S. A.; Oulton R. F.; Hamm J. M.; Tsakmakidis K. L. Active Nanoplasmonic Metamaterials. Nat. Mater. 2012, 11, 573-584

(6) Kauranen M.; Zayats A. V. Nonlinear Plasmonics. Nature 2012, 6, 637-642

(7) Butet J.; Brevet P. F.; Martin O. J. F. Optical Second Harmonic Generation in Plasmonic Nanostructures: From Fundamental Principles to Advanced Applications. ACS Nano 2015, 9, 10545-10562

(8) Yu D.; Wing-Wah V. Controlled Synthesis of Monodisperse Silver Nanocubes in Water. J. Am. Chem. Soc. 2004, 126, 13200-13201

(9) Zhang Q.; Li W.; Moran C.; Zeng J.; Chen J.; Wen L.-P.; Xia Y. Seed-Mediated Synthesis of Ag Nanocubes with Controllable Edge Lengths in the Range of 30-200 nm and Comparison of their Optical Properties. J. Am. Chem. Soc. 2010, 132, 11372-11373

(10) Boltasseva A.; Shalaev V. M. Fabrication of Optical Negative-Index Metamaterials: Recent Advances and Outlook. Metamaterials 2008, 2, 1-17

(11) Ray P. C. Size and Shape Dependent Second Order Nonlinear Optical Properties of Nanomaterials and their Applications. Chem. Rev. 2010, 110, 5332-5365

(12) Zhang K.; Huang Z.-L.; Dai H.-W.; Ma Z.-W.; Han J.-B.; Gong H.-M.; Han Y.-B. Surface Plasmon Enhanced Third-Order Optical Nonlinearity of Silver Nanocubes. Opt. Mat. Express 2015, 5, 2648-2654

(13) Boyd R. M. Nonlinear Optics; Academic Press, San Diego, 1992

(14) Butet J.; Bachelier G.; Russier-Antoine I.; Jonin Ch.; Benichou E.; Brevet P. F. Interference between Selected Dipoles and Octupoles in the Optical Second Harmonic Generation from Spherical Gold Nanoparticles. Phys. Rev. Lett. 2010, 105, 077401

(15) Butet J.; Martin O. J. F. Nonlinear Plasmonic Nanorulers. ACS Nano 2014, 8, 49314939

(16) Smirnova D.; Kivshar Y. S. Multipolar Nonlinear Nanophotonics. Optica 2016, 3, 12411255

(17) Butet J.; Russier-Antoine I.; Jonin Ch.; Lascoux N.; Benichou E.; Brevet P. F. Sensing with Multipolar Second Harmonic Generation from Spherical Metallic Nanoparticles. Nano Lett. 2012, 12, 1697-1701 
(18) Nappa J.; Revillod G.; Russier-Antoine I.; Benichou E.; Jonin Ch.; Brevet P. F. Electric Dipole Origin of the Second Harmonic Generation of Small Metallic Particles. Phys. Rev $B$ 2005, 71, 165407

(19) Zhou F.; Li Z.-Y.; Liu Y.; Xia Y. Quantitative Analysis of Dipole and Quadrupole Excitation in the Surface Plasmon Resonance of Metal Nanoparticles. J. Phys. Chem. C 2008, 112, 20233-20240

(20) El Harfouch Y.; Benichou E.; Bertorelle F.; Russier-Antoine I.; Jonin Ch.; Lascoux N.; Brevet P. F. Hyper Rayleigh Scattering from Gold Nanorods. J. Phys. Chem. C 2014, $118,609-616$

(21) Singh A.; Lehoux A.; Remita H.; Zyss J.; Ledoux-Rak I. Second Harmonic Response of Gold Nanorods: A Strong Enhancement with the Aspect Ratio. J. Phys. Chem. Lett. 2013, 4, 3958-3961

(22) Russier-Antoine I.; Duboisset J.; Bachelier G.; Benichou E.; Jonin Ch.; Del Fatti N.; Vallée F.; Sánchez-Iglesias A.; Pastoriza-Santos I.; Liz-Marzan L. M. ; Brevet P. F. Symmetry Cancellations in the Quadratic Hyperpolarizability of Non-Centrosymmetric Gold Decahedra. J. Phys. Chem. Lett. 2010, 1, 874-880

(23) Fomichev S. V.; Bratkovsky A. M.; Unified Hydrodynamic Approach to Laser Field Plasmon Excitations in Metal Nanoparticles with General Shape. Phys. Rev. B 2013, 88, 045433

(24) Bachelier G.; Butet J.; Russier-Antoine I.; Jonin Ch.; Benichou E.; Brevet P. F. Origin of Optical Second-Harmonic Generation in Spherical Gold Nanoparticles: Local Surface and Nonlocal Bulk Contributions. Phys. Rev. B 2010, 82, 235403

(25) Skrabalak S. E.; Au L.; Li X.; Xia Y. Facile Synthesis of Ag Nanocubes and Au Nanocages. Nat. Protoc. 2007, 2, 2182-2190

(26) Ahamad N.; Bottomley A.; Ianoul A. Optimizing Refractive Index Sensitivity of Supported Silver Nanocube Monolayers. J. Phys. Chem. C 2012, 116, 185-192

(27) Das R.; Sarkar S. Optical Properties of Silver Nanocubes. Opt. Mat. 2015, 48, 203-208

(28) Clays K.; Persoons A. Hyper Rayleigh Scattering in Solution. Phys. Rev. Lett. 1991, 66, 2980-2983

(29) Clays K.; Persoons A. Hyper Rayleigh Scattering in Solution. Rev. Sci. Instrum. 1992, $63,3285-3289$

(30) Duboisset J.; Matar G.; Russier-Antoine I.; Benichou E.; Bachelier G.; Jonin Ch.; Ficheux D.; Besson F.; Brevet P. F. First Hyperpolarizability of the Natural Aromatic Amino Acids Tryptophan, Tyrosine and Phenylalanine and the Tripeptide LysineTryptophan-Lysine Determined by Hyper Rayleigh Scattering. J. Phys. Chem. B 2010, $114,13861-13865$

(31) Russier-Antoine I.; Benichou E.; Bachelier G.; Jonin Ch.; Brevet P. F. Multipolar Contributions of the Second Harmonic Generation from Silver and Gold Nanoparticles. J. Phys. Chem. C 2007, 111, 9044-9048 
(32) Kuzyk M. G.; Singer K. D.; Stegeman G.I. Theory of Molecular Nonlinear Optics. Adv. Opt. Phot. 2013, 5, 4-82

(33) Campo J.; Painelli A.; Terenziani F.; Van Regemorter T.; Beljonne D.; Goovaerts E.; Wenseleers W. First Hyperpolarizability Dispersion of the Octupolar Molecule Crystal Violet: Multiple Resonances and Vibrational and Solvation Effects. J. Am. Chem., Soc. 2010, 132, 16467-16478

(34) Zyss J. Molecular Engineering Implications of Rotational Invariance in Quadratic Nonlinear Optics: From Dipolar to Octupolar Molecules and Materials. J. Chem. Phys. 1993, 98, 6583-6599

(35) Brasselet S.; Zyss J. Multipolar Molecules and Multipolar Fields: Probing and Controlling the Tensorial Nature of Nonlinear Molecular Media. J. Opt. Soc. Am. B 1998, $15,257-288$

(36) Forestiere C.; Capretti A.; Miano G. Surface Integral Method for Second Harmonic Generation in Metal Nanoparticles Including both Local-Surface and Nonlocal-Bulk Sources. J. Opt. Soc. Am. B 2013, 30, 2355-2364

(37) Johnson P. B.; Christy R. W. Optical Constants of the Noble Metals. Phys. Rev. B 1972, $6,4370-4379$

(38) Dadap J. I.; Shan J.; Eisenthal K. B.; Heinz T. F. Second Harmonic Rayleigh Scattering from a Sphere of Centrosymmetric Material. Phys. Rev. Lett. 1999, 83, 4045-4048

(39) Raziman T. V.; Martin O. J. F. Polarisation Charges and Scattering Behaviour of Realistically Rounded Plasmonic Nanostructures. Opt. Express 2013, 21, 21500- 21507 